\title{
UM MODELO DE RECONCILIAÇÃO DE DADOS PARA O CUSTEIO
}

\section{BASEADO-EM-ATIVIDADES (ABC)}

\begin{abstract}
Samuel Cogan
Professor Adjunto da UFRJ, Mestre e Doutor em Engenharia de Produção (Coppe) e autor dos livros Activity-Based Costing $(A B C)$ : a poderosa estratégia empresarial, Modelos de $A B C / A B M$ e Gerenciando as percepções nas filas de espera.

E-mail:scogan@uol.com.br
\end{abstract}

RESUMO: A mudança na estrutura dos custos, em função de novas tecnologias de produção, de uma maior diversificação imposta pela competitividade empresarial e da redução dos custos de medição, criou condições para o aparecimento de técnicas de gerenciamento de custos voltadas para a captação mais precisa das despesas indiretas que cada produto consome. Nesse mister destaca-se o Custeio Baseadoem-Atividades/Gerenciamento Baseado-em-Atividades ( $A B C / A B M)$ como uma das técnicas mais importantes surgidas nos últimos anos, que proporciona uma estimativa mais precisa das despesas indiretas reais que incidem em cada produto. Existem, contudo, certos custos cuja obtenção é difícil mesmo com a utilização de técnicas como o ABC. Isso se deve não só pela impossibilidade, em alguns casos, de serem determinados com adequada exatidão, como, em outros, pelo fato de o investimento na sua medição ultrapassar o benefício a ser obtido com ele. Nesse sentido, o presente trabalho desenvolve, para o fluxo de custos de uma empresa, inspirado na reconciliação de dados utilizada nos processos das plantas químicas, um modelo para a determinação de todos os custos indiretos, partindo do conhecimento preciso de alguns dos custos que compõem o citado fluxo de custos. 0 modelo desenvolvido utiliza programação matemática não-linear.

ABSTRACT: Cost structure changes, due to new production technologies, to major diversification needed because of business competitivity, and to cost reduction, have motivated the development programmes of new cost management - techniques that allocate indirect costs to each product more precisely. In this context, Activity-Based Costing/ActivityBased Management ( $A B C / A B M)$ is considered as one of the most important techniques that have emerged in the last few years to provide accurate estimates for indirect expenses that each product consumes. Nevertheless, some difficulties to obtain some costs remain, even when using ABC. This happens not only due to the impossibility to measure costs with adequate accuracy, but also due to the fact that the investments made to measure some costs exceed the benefits to be obtained. In order to address this problem, the present work provides a business cost flow framework utilizing data reconciliation used in chemical process networks to determine indirect costs, incorporating accurate information concerning some of the costs. The model developed utilizes non-linear programming. 


\section{INTRODUÇÃO}

O objetivo do presente trabalho é criar um modelo baseado na reconciliação de dados que, conjugado ao custeio $\mathrm{ABC}$, permita que se determinem os custos indiretos que cada produto consome para os casos em que existem custos de difícil mensuração. A solução do modelo desenvolvido é viabilizada pela associação da reconciliação dos dados obtidos de um modelo $\mathrm{ABC}$ com a técnica de programação matemática não-linear (Cogan, 1995).

A importância do tema em referência deve-se à drástica alteração da estrutura de custos dos produtos/serviços nas últimas décadas. A parcela de despesas indiretas, que, no passado, apresentava valores pequenos em relação aos custos totais, cresceu sobremodo. Isso ocorreu devido à conjugação de novas técnicas de gerenciamento, novas tecnologias de produção, estratégias de marketing com uma diversificação de produtos cada vez maior etc.

\section{A parcela de despesas indiretas, que, no passado, apresentava valores pequenos em relação aos custos totais, cresceu sobremodo.}

Nessas condições, os rateios simplistas pregados pela contabilidade de custos tradicional não mais atendem aos reclamos dessa nova era, sob o risco de deixarem as empresas com custos distorcidos, o que tornaria as decisões gerenciais, enfocando a rentabilidade dos produtos/serviços, um mero exercício de "intuição". Assim, nos últimos anos, surgiu e vem se espalhando pelas empresas de todo o mundo uma forma mais avançada de determinação dessas despesas indiretas por meio do custeio baseado nas atividades que consomem recursos (Cooper e Kaplan, 1991).

O presente trabalho considera a inconveniência da existência de custos de difícil medição e o reflexo que esses custos podem trazer aos resultados finais mesmo com a utilização do $\mathrm{ABC}$. Esses custos podem apresentar dificuldades de mensuração não só pela impossibilidade técnica de medição, como também outros casos existem em que, embora sejam tecnicamente viáveis de serem obtidos, o investimento realizado pode não ter retorno. Considerando isso, este trabalho cria, para os esquemas $\mathrm{ABC}$ desenvolvidos, um modelo de reconciliação de dados que se utiliza dos resultados obtidos por direcionadores de custos precisos (alocação direta ou alocação com alta base causal). Os custos de difícil mensuração serão, então, obtidos por meio da conjugação do sistema $\mathrm{ABC}$ com a técnica de reconciliação de dados, técnica essa já utilizada nos fluxos de massas dos processos das plantas químicas.

\section{POR QUE RECONCILIAÇÃO DE DADOS NA GESTÃO DE CUSTOS}

\section{Mudanças nas estruturas dos custos}

O rápido e grande avanço observado no ambiente da produção, por meio do surgimento de novas estratégias de manufatura, impôs mudanças profundas na forma de se produzir. Uma das técnicas mais atingidas por essas mudanças é a que se refere ao gerenciamento de custos.

Até os anos 70, as parcelas diretas de mão-de-obra e material respondiam pela quase totalidade dos custos totais. Outras despesas indiretas, como qualidade, controle da produção, compras etc., representavam uma pequena proporção. Em decorrência disso, os métodos tradicionais de alocação dos custos indiretos, por uma questão de simplificação, recomendavam o rateio de tais despesas de acordo com critérios baseados nos volumes do mix de produtos.

Entretanto, a estrutura de custo dos produtos vem mudando de tal forma que as despesas indiretas, antes da ordem de $5 \%$ dos custos, hoje já alcançam valores médios superiores a $35 \%$, havendo casos de empresas com elevada intensidade de automatização nas quais as despesas podem atingir $70 \%$ dos custos. Por outro lado, no passado, os custos de medição das despesas eram elevados, e a diversificação dos produtos, pequena. Hoje, com o avanço tecnológico obtido, os custos de medição estão mais compatíveis, permitindo que se possa caminhar em direção a uma apuração mais precisa dos custos (Cogan, 1994). Nos tempos atuais, também a diversificação de produtos e serviços vem crescendo com a tendência de se procurar atingir uma operação que atenda aos clientes com produtos/serviços personalizados. Essas considerações se somam para que se afirme que o custeio tradicional se tornou bastante inadequado - novas estruturas de custeios precisam, portanto, ser pesquisadas e desenvolvidas.

Esse desafio no gerenciamento de custos deu origem ao surgimento de um certo número de novos conceitos, sendo que o mais bem-sucedido tem sido o Custeio Baseado-em-Atividades (ABC). A premissa básica do $\mathrm{ABC}$ é tratar os custos indiretos como se 
fossem diretos, por meio da análise de suas atividades. Como conseqüência do $\mathrm{ABC}$, surgiu a possibilidade de se melhorar/reduzir os custos das atividades dos processos que atravessam transversalmente a empresa pela utilização do Gerenciamento Baseado-emAtividades (ABM).

$\mathrm{O}$ sistema $\mathrm{ABC}$ procura atribuir aos produtos individuais, além das despesas diretas que incidem em cada produto/peça, as despesas indiretas como se fossem diretas - quer sejam elas acumuladas em atividades de lotes/ordens de produção, atividades de suporte dos produtos ou atividades para suporte das facilidades usando, para tanto, bases de atribuição das despesas, denominadas direcionadores de custos, que procuram refletir a parcela de demanda que deverá incidir sobre cada produto per se.

\section{A reconciliação de dados}

O status de uma planta química de processo operacional é definido pelos resultados obtidos com os instrumentos que medem seu desempenho. O controle e a operação dessa planta consistem em manter esse estado dentro de certos limites. Entretanto, os dados de medição realizados na citada planta são obtidos utilizando instrumentos que são essencialmente imprecisos e que, em decorrência disso, provavelmente não irão satisfazer os equilíbrios reais de massa. Os dados precisam, portanto, ser reconciliados, levando-se em conta esses fatos a fim de que se possa obter uma imagem verdadeira do estado do referido processo.

Cabe ao staff operacional a função de manter a planta operacional dentro de limites bem definidos. Entretanto, os dados medidos na planta da forma como são feitos podem não ser confiáveis, em virtude da utilização, muitas vezes, de instrumentos de medição imprecisos. No caso de plantas químicas operacionais, Hlavaceck (1977) considera a existência de três tipos de erros. O primeiro se refere aos ajustes dos equilíbrios de massa sujeitos a pequenos erros randômicos, o segundo alude aos ajustes dos equilíbrios de massa sujeitos a erros grosseiros e o terceiro tipo de erro relaciona-se à escolha dos pontos de medição. A questão da reconciliação é, então, usada para denotar o problema de predizer o valor dos dados, tanto dos que foram medidos quanto dos que não foram medidos.

A reconciliação dos equilíbrios de massa nos processos de plantas operacionais consiste, então, em predizer os valores dos fluxos de massas em todas as correntes de uma dada planta de processo, na qual somente um conjunto de fluxos é medido. Isso é chamado de problema de reconciliação dos equilíbrios de massa. Em primeiro lugar, as necessidades requeridas pelo sistema são examinadas. O problema é, então, descri- to em termos matemáticos e duas técnicas principais são utilizadas para resolvê-lo: a programação linear e a programação quadrática.

Uma dificuldade adicional é causada pelo fato de que nem todas as variáveis são medidas tendo em vista considerações de custos ou mesmo impossibilidade técnica. Dessa forma, torna-se necessário ajustar as variáveis medidas e, se possível, estimar as variáveis não medidas de tal forma que elas satisfaçam às restrições de equilíbrio, processo esse que é conhecido como problema de reconciliação de dados (Tamhano e Mah, 1985).

\section{Os rateios simplistas pregados pela contabilidade de custos tradicional não mais atendem aos reclamos dessa nova era, sob o risco de deixarem as empresas com custos distorcidos.}

No custeio $\mathrm{ABC}$, por seu turno, quando se analisa a distribuição de custos (Ostrenga et al., 1993), a primeira ação é verificar se existem custos dedicados integralmente a atividades e/ou produtos, ação essa conhecida por alocação direta. No caso de não poder enquadrar-se na alocação direta, a segunda ação volta-se para uma alocação com base causal ou de atividade. E, em última instância, não ocorrendo as duas situações anteriores, a terceira ação é a técnica do enquadramento baseado em volume.

No que se refere aos custos com alocação de base causal, alguns problemas existem que podem dificultar a eleição do melhor determinante de atividade/ direcionador de custos, ou seja, muitas vezes se podem ter escolhido direcionadores de alto relacionamento causal que, entretanto, apresentam alta dificuldade de medição, baixa disponibilidade de dados e alta dificuldade de quantificação. Por outro lado, certos direcionadores podem ter sido escolhidos por apresentarem facilidades de medição, disponibilidade de dados e por serem quantificáveis e, no entanto, podem apresentar uma baixa relação com as causas básicas.

$\mathrm{O}$ explanado acima ilustra os problemas que ocorrem na prática e que podem dificultar a identificação precisa dos custos mesmo com o custeio ABC, daí os esforços de pesquisas para o aprimoramento dessa sistemática. 
Dessa forma, e à semelhança do que acontece numa planta química, pode-se visualizar um modelo de um sistema de custos como uma rede pela qual fluem os custos. Esses custos estão sujeitos a certa quantidade de restrições e, igualmente ao apontado nos trabalhos de reconciliação de dados nas plantas químicas, existem dificuldades na mensuração de alguns deles. Assim, neste trabalho será apresentada uma tentativa de solução para essa problemática, ou seja, um modelo de reconciliação de dados para o ABC previamente calculado que, valendo-se das técnicas de programação matemática, busca chegar aos valores de custos naqueles pontos do sistema em que a informação disponível é pobre ou inviável de se melhorar, mantendo-se, contudo, os equilíbrios da fluidez de custos por toda a rede.

\section{DESENVOLVIMENTO DO MODELO}

A fim de descrever o problema matematicamente, considerou-se um processo constituído de " $n$ " correntes, representado por uma rede de "k" nós. Do número total de correntes "n", somente "m" são medidas. A precisão das medidas é indicada por "m" valores de custos.
A função-objetivo a ser minimizada é (Gorczynski, 1985):

$$
\begin{aligned}
& \text { Minimizar [função de (R, W)] } \\
& \text { onde } \quad \mathbf{R}=\mathbf{V}-\mathbf{B X} \text { ("m" relações de medições) } \\
& \text { sujeito a } \mathbf{M X}=\mathbf{0} \\
& \mathbf{X} \geq \mathbf{0} \\
& \text { com } \quad \mathbf{n}>\mathbf{k} \\
& \mathbf{m}>\mathbf{n}-\mathbf{k}
\end{aligned}
$$

Figura 1 - Sistema "ABC”

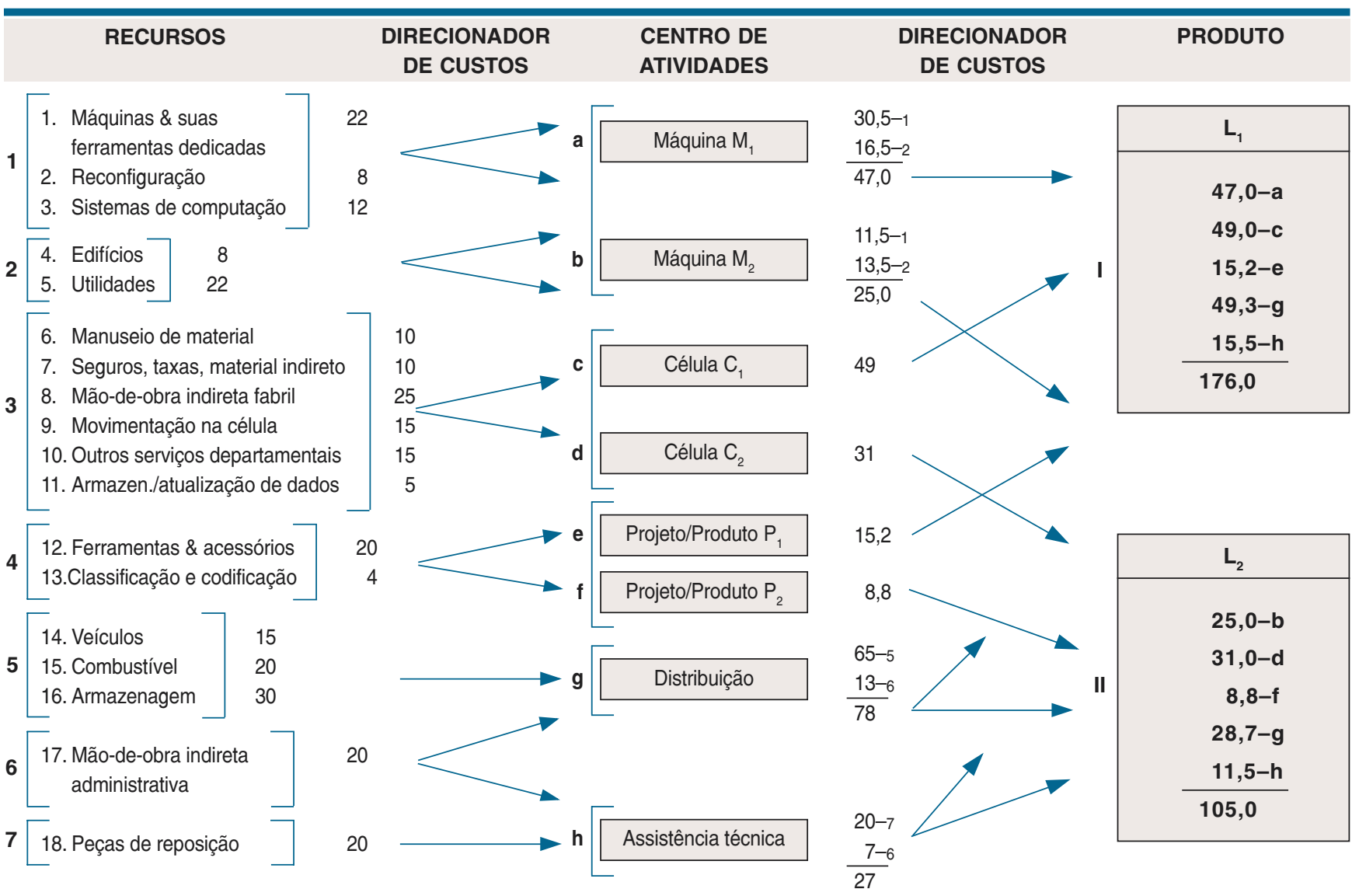




\section{EXEMPLO PRÁTICO}

\section{Construção do modelo ABC}

Tomemos o seguinte exemplo de modelo ABC, cujo diagrama esquemático é apresentado na Figura 1. Ele espelha a fabricação, a entrega e a assistência pósvenda de dois produtos $\left(\underline{\mathrm{P}}_{1}\right.$ e $\left.\underline{\mathrm{P}}_{2}\right)$ que são fabricados em células de produção separadas $\left(\underline{\mathrm{C}}_{1}\right.$ para o produto $\underline{\mathrm{P}}_{1} \mathrm{e}$ $\underline{\mathrm{C}}_{2}$ para o produto $\left.\underline{\mathrm{P}}_{2}\right)$. Considera-se ainda que a empresa produz mensalmente o lote $\underline{L}_{1}$, que se refere ao produto $\underline{\mathrm{P}}_{1}$, e o lote $\underline{\mathrm{L}}_{2}$, que se refere ao produto $\underline{\mathrm{P}}_{2}$. Existem duas máquinas, $\underline{\mathrm{M}}_{1}$, situada na célula $\underline{\mathrm{C}}_{1}$, e $\underline{\mathrm{M}}_{2}$, que está localizada na célula $\underline{\mathrm{C}}_{2}$. O lote $\underline{\mathrm{L}}_{1}$ opera tãosomente na célula $\underline{C}_{1}$, enquanto o lote $\underline{L}_{2}$ somente trabalha na célula $\underline{\mathrm{C}}_{2}$. Conforme pode ser observado nesse diagrama, o modelo apresenta oito centros distintos de acumulação de custos por atividades: um para cada uma das máquinas; um para cada uma das células; um para cada um dos projetos de produtos; outro para os recursos de distribuição e outro, ainda, para as despesas de assistência técnica. Observe-se, ainda, que os valores anotados no diagrama em referência estão em milhares de dólares.

Esse diagrama mostra despesas que usualmente ocorrem numa empresa, num total de 18 . Nos dois primeiros centros de acumulação de custos por atividades (um para cada máquina), são canalizados os recursos correspondentes aos valores registrados nos livros contábeis da empresa referentes à depreciação de cada "máquina e suas ferramentas e acessórios dedicados". Também aí incidem uma parte do custo da "reconfiguração" da citada planta em células de produção (a amortização para o período) e uma porção do capital gasto em "sistemas de computação" (a amortização para o período). No caso dos recursos "reconfiguração" e "sistemas de computação", torna-se necessário, num primeiro estágio, apurar os custos que incidem em cada uma das máquinas, para o que se utiliza um direcionador de custos baseado nos custos de cada máquina. No exemplo, os livros contábeis da empresa indicaram os seguintes dados de depreciação: US\$ 16 mil para $\underline{\mathrm{M}}_{1}$ e US\$ 6 mil para $\underline{\mathrm{M}}_{2}$ (correspondentes à despesa 1 , "Máquinas \& suas ferramentas dedicadas", no valor total de US\$ $22 \mathrm{mil}$ ). Para as despesas 2 e 3, o valor de US\$ 8 mil somado a US\$ 12 mil será aplicado aos centros de atividades $\underline{\mathrm{M}}_{1}$ e $\underline{\mathrm{M}}_{2}$ na mesma proporção de US\$ 16 mil e US\$ 6 mil (direcionador baseado no custo das máquinas). Assim, os valores para as despesas 2 e 3 serão de US\$ 14,5 mil para $\underline{\mathrm{M}}_{1}$ e US\$ 5,5 mil para $\underline{\mathrm{M}}_{2}$. Os valores totais para o recurso 1 ("Máquinas \& suas ferramentas dedicadas", "Reconfiguração" e "Sistemas de computação") serão de US\$ 30,5 mil para $\underline{M}_{1}$ e US\$ 11,5 mil para $\underline{\mathrm{M}}_{2}$. O recurso 2 , que engloba as despesas 4 e 5
("Edifícios" e "Utilidades"), agrupa custos que necessariamente ocorrem no período em conseqüência de "aluguel dos prédios ou respectivas depreciações", "serviços de utilidades, segurança e terreno" etc.

\section{O rápido e grande avanço observado no ambiente da produção, por meio de novas estratégias de manufatura, impôs mudanças profundas na forma de se produzir.}

Como esses custos ocorrem para o total das facilidades existentes, eles são então alocados para cada máquina com base na área relativa ocupada no chãode-fábrica. Foi, então, utilizado um direcionador de custos de primeiro estágio, baseado nas áreas ocupadas, sendo que $55 \%$ se referem a $\underline{\mathrm{M}}_{1}$ e $45 \%$ a $\underline{\mathrm{M}}_{2}$, obtendo-se, então, US\$16,5 mil para $\underline{\mathrm{M}}_{1}$ e US\$13,5 mil para $\underline{\mathrm{M}}_{2}$. Num segundo estágio, os totais de $\underline{\mathrm{M}}_{1} \mathrm{e}$ $\underline{\mathrm{M}}_{2}$ serão distribuídos em sua totalidade, respectivamente para as células $\underline{L}_{1}$ (US\$ 47 mil) e $\underline{L}_{2}$ (US\$ 25 mil), que são os objetos dos custos.

A "Mão-de-obra indireta fabril", que apóia a produção, também tem suas despesas carregadas diretamente nas respectivas células para as quais estão dando suporte. Esses recursos são canalizados para o terceiro e quatro centros de custos por atividade que está sendo considerada - centros de atividades para cada célula. Em seguida, esses custos são atribuídos diretamente aos lotes $\underline{L}_{1}$ e $\underline{L}_{2}$, à semelhança do que se fez anteriormente com os centros de atividades de máquinas. Da mesma maneira, nesses centros de custos (de cada célula), incidem as despesas de "Manuseio de material" e "Movimentação na célula". É conveniente notar que essas despesas se comportam como se fossem custos diretos apropriados na célula. "Seguros" e "Taxas" são proporcionais aos valores dos bens e podem ser apropriados às células com base no valor total dos bens contidos nas células. "Suprimentos" e "Material indireto" também se comportam como se os custos fossem diretos, uma vez que seu uso ocorre dentro de cada célula. Da mesma forma, os custos correspondentes a "Outros serviços departamentais" e os relacionados a "Armazenamento, atualização e recuperação de dados" também são alocados diretamente nas células. A homogeneidade dos componentes num centro 
de custos é um requerimento que é facilmente encontrado num sistema de manufatura celular devido à similaridade dos produtos numa família de produtos. O direcionador de custos deverá ter uma relação causal com o consumo real dos recursos de um centro de custos por atividades. Assim, para o centro de custos de cada célula, também se dispensa a necessidade do direcionador de custos, pois cada célula descarrega seus recursos no lote respectivo, como já comentado, perfazendo os valores estimados de US\$ 49 mil e US\$ 31 mil, respectivamente.

\section{Torna-se necessário ajustar as variáveis medidas e, se possível, estimar as variáveis não medidas de tal forma que elas satisfaçam às restrições de equilíbrio.}

O quinto e o sexto centros de acumulação de custos por atividades - centro de custos para cada projeto de produto - agregam as despesas especificamente incorridas no que se refere a "Ferramentas e acessórios" e também os gastos de "Classificação e codificação das peças". Nessa última sigla relacionam-se as despesas envolvidas na análise das características do produto e no estabelecimento de um código para cada produto, de tal forma que informações concernentes a elas possam ser armazenadas num arquivo de dados. Esses e os custos específicos de "Ferramentas e acessórios" desenvolvidos para cada componente são acumulados em centros de custos por atividades, um para cada produto, e apropriados também diretamente a cada lote. Observe-se que esses recursos referem-se ao investimento a ser realizado, o qual, por seu turno, terá de ser amortizado mensalmente durante todo o ciclo de vida do produto. Isso deverá perfazer os valores de US\$ 15,2 mil e US $\$ 8,8$ mil, respectivamente para o projeto de $\underline{\mathrm{P}}_{1}$ e para o projeto de $\underline{\mathrm{P}}_{2}$. Esses valores se aplicam diretamente a cada lote, como também ocorreu com os centros de atividades anteriores.

No recurso 5, estão englobadas as despesas de "Veículos", "Combustível" e "Armazenagem", no valor total de US\$ 65 mil, que se aplicam diretamente ao centro de atividades "Distribuição". As despesas denominadas "Veículos" e "Combustível" referem-se às despesas de transporte dos lotes de mercadorias, enquanto as despesas de "Armazenagem" correspondem aos custos totais envolvidos no dia-a-dia de um depó- sito de produtos acabados, acrescidas das despesas de preparação dos pedidos, da seleção, do empacotamento e do embarque destes. O recurso 7, "Peças de reposição", refere-se às peças que são gastas na assistência técnica e aplica-se diretamente ao centro de atividades "Assistência técnica". O recurso 6, "Mão-de-obra indireta administrativa", aplica-se tanto ao centro de atividades "Distribuição" quanto ao de "Assistência técnica". Para tanto, torna-se necessária a utilização de um direcionador de primeiro estágio - no caso, o direcionador usado foi a previsão de mão-de-obra a ser efetivamente aplicada nas atividades de cada centro. Dessa forma, chegou-se aos valores de US\$ 13 mil e US\$ 7 mil para cada centro de atividades. Como pode ser visto no diagrama, o centro de atividades "Distribuição" acumula um total de US\$ 78 mil, e o centro de atividades de "Assistência técnica", o total de US\$ 27 mil. O valor acumulado no centro "Distribuição" será atribuído aos dois objetos de custos segundo um direcionador de segundo estágio - no caso, o escolhido foi "as porcentagens dos valores de vendas", tendo-se partido do pressuposto de que os dois lotes apresentam tamanhos e valores unitários semelhantes e são entregues a clientes situados em distâncias aproximadamente iguais, além de apresentarem custos diretos semelhantes. Assim, obtiveram-se os valores de US\$ 49,3 mil e US\$28,7 mil, respectivamente para os lotes 1 e 2 . No que se refere aos recursos acumulados no centro de atividades "Assistência técnica", a previsão é que essas despesas serão distribuídas pelos dois objetos de custo de acordo com as despesas efetivamente registradas para cada reclamação de cliente e por lote. Chega-se, então, a US\$15,5 mil e US\$11,5 mil. Dessa forma, foram obtidos o valor total de US\$ 176 mil para $\underline{L}_{1}$ e o de US\$105 mil para $\underline{L}_{2}$.

\section{Modelo de reconciliação de dados aplicado ao modelo ABC apresentado}

Observe o leitor que a Figura 2 reproduz a Figura 1 sob a forma de um diagrama de flechas (" $n=25$ " correntes, das quais " $\mathrm{m}$ " são medidas e " $\mathrm{k}=11$ ", em que "k" são nós). A metodologia de reconciliação de dados a ser aplicada a esse sistema $\mathrm{ABC}$ partiu do pressuposto de que as alocações dos centros de atividades "Distribuição" e "Assistência técnica" para os dois objetos de custos (lotes $\underline{L}_{1}$ e $\underline{L}_{2}$ ) não eram confiáveis no que se refere à precisão dos direcionadores usados. Supôs-se, ainda, que os demais cálculos são precisos e que serão, então, os valores a serem considerados no novo modelo. Espera-se determinar os valores dos demais. Além disso, esse novo modelo vai também reconciliar os valores fornecidos, ou seja, aqueles considerados precisos no modelo ABC res- 
pectivo. Esse sistema deve atender a uma série de restrições, como o equilíbrio em cada nó. A Figura 2 mostra a existência de 11 nós e 25 correntes (de $X_{1}$ a $X_{25}$ ). Pode-se, então, construir o seguinte modelo, constituído das equações:

1) $X_{1}-X_{8}-X_{9}=0$

[equilíbrio no nó 1]

2) $X_{2}-X_{10}-X_{11}=0$ [equilíbrio no nó 2]

3) $X_{3}-X_{12}-X_{13}=0$ [equilíbrio no nó 3]

4) $X_{4}-X_{14}-X_{15}=0$ [equilíbrio no nó 4]

5) $X_{6}-X_{16}-X_{17}=0$ [equilíbrio no nó 5]

6) $X_{8}+X_{10}-X_{18}=0$ [equilíbrio no nó 6]

7) $X_{9}+X_{11}-X_{19}=0$ [equilíbrio no nó 7]

8) $X_{5}+X_{16}-X_{20}-X_{21}=0$ [equilíbrio no nó 8]

9) $X_{17}+X_{7}-X_{22}-X_{23}=0$ [equilíbrio no nó 9]

10) $X_{18}+X_{12}+X_{14}+X_{20}+X_{22}-X_{24}=0$

[equilíbrio no nó 10]

11) $X_{19}+X_{13}+X_{15}+X_{21}+X_{23}-X_{25}=0$

[equilíbrio no nó 11]
Outras restrições a serem atendidas são as de que o valor de cada $\mathrm{X}_{\mathrm{i}}$ deverá ser positivo. Então podese escrever:
12) $X_{1}>0$
13) $X_{2}>0$
14) $X_{3}>0$
15) $\mathbf{X}_{4}>\mathbf{0}$
16) $X_{5}>0$
17) $X_{6}>0$
18) $\mathbf{X}_{7}>\mathbf{0}$
19) $\mathbf{X}_{8}>\mathbf{0}$
20) $X_{9}>0$
21) $X_{10}>0$
22) $X_{11}>0$
23) $X_{12}>0$
24) $X_{13}>0$
25) $X_{14}>0$
26) $X_{15}>0$
27) $X_{16}>0$
28) $\mathbf{X}_{17}>\mathbf{0}$
29) $X_{18}>0$
30) $\mathbf{X}_{19}>0$
31) $\mathbf{X}_{20}>\mathbf{0}$
32) $X_{21}>0$
33) $X_{22}>0$
34) $X_{23}>0$
35) $\mathbf{X}_{24}>\mathbf{0}$
36) $X_{25}>0$

Além disso, os recursos devem somar os valores registrados nos livros da companhia, para as despesas:

37) $X_{1}+X_{2}+X_{3}+X_{4}+X_{5}+X_{6}+X_{7}=281$

Figura 2 - Modelo de reconciliação de dados aplicado ao modelo ABC apresentado

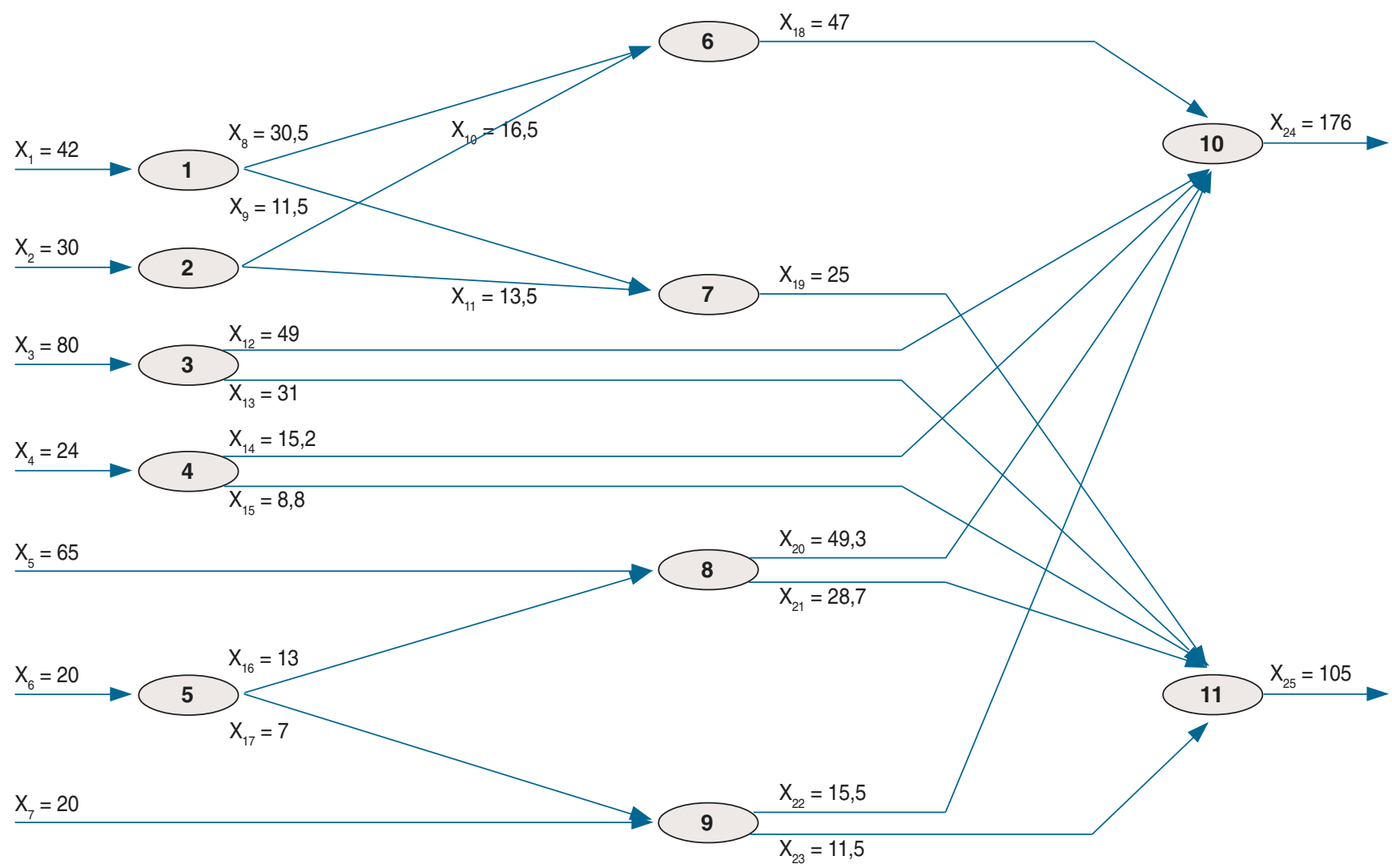


Finalmente, a equação de otimização do sistema é:

$$
\text { 38) } \begin{aligned}
\text { MIN = } & \left(X_{1}-42\right)^{2}+\left(X_{2}-30\right)^{2}+\left(X_{3}-80\right)^{2}+\left(X_{4}-24\right)^{2}+\left(X_{5}-65\right)^{2}+\left(X_{6}-20\right)^{2}+\left(X_{7}-20\right)^{2}+\left(X_{8}-30,5\right)^{2}+\left(X_{9}-11,5\right)^{2} \\
& +\left(X_{10}-16,5\right)^{2}+\left(X_{11}-13,5\right)^{2}+\left(X_{12}-49\right)^{2}+\left(X_{13}-31\right)^{2}+\left(X_{14}-15,2\right)^{2}+\left(X_{15}-8,8\right)^{2}+\left(X_{16}-13\right)^{2}
\end{aligned}
$$

Assim, a técnica da programação matemática nãolinear minimizará os quadrados das diferenças entre os resultados reconciliados de cada $\mathrm{X}_{\mathrm{i}}$ com seus valores considerados precisos obtidos no modelo $\mathrm{ABC}$ em referência e respeitando as demais restrições das equações de 1 a 37.

A solução dessas 38 equações pode ser obtida rapidamente por meio da utilização de um aplicativo como o GINO (General Interactive Optimizer) (Liebman et al., 1984). O resultado obtido é, então, o seguinte:

$$
\begin{array}{llll}
X_{1}=42,00 & X_{2}=30,00 & X_{3}=80,00 & X_{4}=24,00 \\
X_{5}=65,00 & X_{6}=20,00 & X_{7}=20,00 & X_{8}=30,50 \\
X_{9}=11,50 & X_{10}=16,50 & X_{11}=13,50 & X_{12}=49,00 \\
X_{13}=31,00 & X_{14}=15,20 & X_{15}=8,80 & X_{16}=13,00 \\
X_{17}=7,00 & X_{18}=47,00 & X_{19}=25,00 & X_{20}=42,70 \\
X_{21}=35,30 & X_{22}=4,53 & X_{23}=22,47 & X_{24}=158,42 \\
X_{25}=122,58 & & &
\end{array}
$$

Pode-se, então, notar que o modelo de reconciliação de dados apresentou novos valores para $\mathrm{X}_{22}, \mathrm{X}_{23}$, $\mathrm{X}_{24}$ e $\mathrm{X}_{25}$.

\section{CONCLUSÕES}

A principal contribuição deste trabalho consiste no desenvolvimento de um modelo baseado no conceito de reconciliação de dados para a determinação dos custos indiretos consumidos pelos produtos ou serviços - objetos dos custos. Esse modelo se aplica conjugado aos diagramas esquemáticos obtidos por meio da aplicação do ABC/ABM a cada caso.

Como se sabe, o ABC representa um avanço em relação às práticas tradicionais de custeio para obtenção de custos indiretos dos produtos de forma mais precisa. É usual, todavia, a existência de alguns custos de difícil mensuração, quer por serem impossíveis de ser obtidos na prática ou por não apresentarem retorno que justifique os investimentos realizados. Assim, o custeio ABC, em alguns casos, pode utilizar direcionadores de custos de baixa relação causal ou baseados em volume quando não existir uma base causal clara ou quando o custo da mensuração for proibitivo em relação ao investimento necessário.

A reconciliação de dados, contudo, fixando-se naqueles direcionadores altamente precisos do $\mathrm{ABC}$ (alocação direta ou alocação com forte base causal) e utilizando-se de programação matemática nãolinear, reconcilia as demais taxas de consumo (correspondentes aos direcionadores de custos do $\mathrm{ABC}$ ), minimizando os erros e mantendo o equilíbrio da rede de custos como um sistema. $\bigcirc$

REFERÊNCIAS BIBLIOGRÁFICAS

COGAN, S. Activity-Based Costing (ABC): a poderosa estratégia empresarial. São Paulo: Pioneira, 1994.

\section{Um modelo de reconciliação de dados para 0} Custeio Baseado-em-Atividades (ABC). Rio de Janeiro, 1995. Tese (Doutorado) - Universidade Federal do Rio de Janeiro, Coppe.

COOPER, R., KAPLAN, R. S. The design of cost management systems: text, cases and readings. Englewood Cliffs: PrenticeHall, 1991.
GORCZYNSKI, E. W. Making the most of your plant data. Proc. Syst. Eng. PSE 85: The use of computers in chemical engineering. Instr. Chem. Engrs. Symposium Series, n.92, 1985.

HLAVACECK, V. Analysis of a complex plant: steady state and transient behavior. Computers in Chemical Engineering, v.1, p.75-100, 1977.
LIEBMAN, J. et al. Applications of modeling and optimization with GINO. USA: The Scientific Press, 1984.

OSTRENGA, M. R. et al. Guia Ernst \& Young para gestão total dos custos. Rio de Janeiro: Record, 1993.

TAMHANO, A. C., MAH, R. S. H. Data reconciliation and gross error detection in chemical process' networks. Technometrics, v.27, n.4, Nov. 1985. 